\title{
Assessment of panobacumab as adjunctive immunotherapy for the treatment of nosocomial Pseudomonas aeruginosa pneumonia
}

\author{
Y.-A. Que • H. Lazar • M. Wolff • B. François • P.-F. Laterre • E. Mercier • \\ J. Garbino • J.-L. Pagani • J.-P. Revelly • E. Mus • A. Perez • M. Tamm • \\ J.-J. Rouby • Q. Lu • J. Chastre • P. Eggimann
}

Received: 25 January 2014 / Accepted: 5 May 2014 /Published online: 24 May 2014

(C) Springer-Verlag Berlin Heidelberg 2014

\begin{abstract}
The fully human anti-lipopolysaccharide (LPS) immunoglobulin M (IgM) monoclonal antibody panobacumab was developed as an adjunctive immunotherapy for the treatment of O11 serotype Pseudomonas aeruginosa infections. We evaluated the potential clinical efficacy of panobacumab in the treatment of nosocomial pneumonia. We performed a post-hoc analysis of a multicenter phase IIa trial (NCT00851435) designed to prospectively evaluate the safety and pharmacokinetics of panobacumab. Patients treated with panobacumab $(n=17)$, including 13 patients receiving the full treatment (three doses of $1.2 \mathrm{mg} / \mathrm{kg}$ ), were compared to 14 patients who did not receive the antibody. Overall, the 17 patients receiving panobacumab were more ill. They were an average of 72 years old [interquartile range (IQR): 64-79] versus an average of 50 years old (IQR: $30-73$ ) $(p=0.024)$ and had Acute Physiology and Chronic Health Evaluation II (APACHE II) scores of 17 (IQR: 16-22) versus 15 (IQR: $10-19)(p=0.043)$. Adjunctive immunotherapy resulted in an improved clinical outcome in the group receiving
\end{abstract}

Y.-A. Que · J.-L. Pagani $\cdot$ J.-P. Revelly $\cdot$ P. Eggimann $(\bowtie)$

Adult Critical Care Service, Centre Hospitalier Universitaire

Vaudois, CHUV, Rue du Bugnon 46, 1011 Lausanne, Switzerland

e-mail: Philippe.Eggimann@chuv.ch

H. Lazar $\cdot$ E. Mus $\cdot$ A. Perez

Kenta Biotech, Schlieren, Switzerland

\section{Wolff}

Réanimation Médicale et Infectieuse, Hopital Bichat-Claude

Bernard, AP-HP, Paris, France

B. François

Intensive Care Unit, CIC-P 0801 Inserm, CHU Dupuytren, Limoges,

France

\section{P.-F. Laterre}

Intensive Care Unit, Saint-Luc University Hospital, UCL, Brussels, Belgium the full three-course panobacumab treatment, with a resolution rate of $85 \%(11 / 13)$ versus $64 \%(9 / 14)(p=0.048)$. The Kaplan-Meier survival curve showed a statistically significantly shorter time to clinical resolution in this group of patients (8.0 [IQR: 7.0-11.5] versus 18.5 [IQR: 8-30] days in those who did not receive the antibody; $p=0.004)$. Panobacumab adjunctive immunotherapy may improve clinical outcome in a shorter time if patients receive the full treatment (three doses). These preliminary results suggest that passive immunotherapy targeting LPS may be a complementary strategy for the treatment of nosocomial O11 P. aeruginosa pneumonia.

\section{Introduction}

Pseudomonas aeruginosa is a recalcitrant, opportunistic Gram-negative bacteria responsible for most nosocomial infections of critically ill patients [1-3]. The development of multidrug resistance makes the treatment of infections caused

E. Mercier

Service de Réanimation Polyvalente and CRICS Network, CHRU de

Tours, Tours, France

J. Garbino

Infectious Diseases, University Hospitals of Geneva, Geneva,

Switzerland

M. Tamm

Pneumonia Clinic, University Hospital Basel, Basel, Switzerland

J.-J. Rouby $\cdot$ Q. Lu

Réanimation Polyvalente, Département d'Anesthésie-Réanimation, Hôpital Pitié-Salpêtrière, Assistance Publique-Hôpitaux de Paris,

UPMC Paris 6, Paris, France

J. Chastre

Service de Réanimation Médicale, Hôpital Pitié-Salpêtrière, AP-HP, Paris, France 
by this pathogen challenging $[4,5]$ and accounts for the persistently high mortality rates observed in recent surveys $[6,7]$, despite aggressive supportive management $[8,9]$. The number of new antimicrobials under clinical development remains extremely limited, and their introduction to the market is disappointingly slow [10], highlighting the urgency for innovative approaches to be developed for the management of multidrug-resistant infections $[11,12]$.

Adjunctive passive immunotherapy specifically targeted at microbial products could be a promising strategy in overcoming this problem. Historically, passive immunotherapy using anti-sera was successfully carried out before the antibiotic era for the management of devastating infectious diseases, such as rabies and diphtheria [13]. The administration of intravenous immunoglobulins as prophylaxis or as combination therapy with antibiotics has shown promising results in treating sepsis $[14,15]$. The development of monoclonal antibodies (MAbs) has improved antibody-based therapies by targeting specific virulence factors on respective pathogens. However, adjunctive immunotherapy targeted at host response mediators has, so far, failed [16]. Therapies combining antimicrobials with MAbs targeted at bacterial targets may potentially result in a more rapid resolution of infections, resulting in reductions in sepsis-related morbidity and mortality [17].

The membrane-bound virulence factor lipopolysaccharide (LPS), expressed by all Gram-negative bacteria like P. aeruginosa, elicits an immunoglobulin M (IgM)-mediated antibody response that takes several days to fully develop, and this delay may increase the risk of death in severe infections [18]. Human monoclonal antibodies directed against P. aeruginosa LPS have demonstrated protection in various settings [19-21]. Panobacumab is a fully human monoclonal antibody of the $\operatorname{IgM} / K$ isotype directed against the LPS Opolysaccharide moiety of $P$. aeruginosa $\mathrm{O} 11$ [22, 23]. $P$. aeruginosa international antigenic scheme serotypes $\mathrm{O} 11$ and $\mathrm{O} 6$ have been reported to be the most prevalent serotypes among epidemiological studies [24, 25]. Strains exhibiting exotoxin $\mathrm{U}$, a potential virulence factor, were frequently serotyped as O11 $[25,26]$. In an experimental model of pneumonia, serotype O11 was found to be associated with increased lung injury [27]. These epidemiological data were confirmed by a recent retrospective cohort study on 123 patients with nosocomial $P$. aeruginosa pneumonia, in which O6 (29\%) and O11 (23\%) were the most prevalent serotypes [28]. In this study, serotype O11 was associated with increased persisting pneumonia and decreased clinical resolution compared to other serotypes. Preliminary data from an open phase IIa study demonstrated the safety of panobacumab in critically ill patients presenting with $P$. aeruginosa $\mathrm{O} 11$ nosocomial pneumonia [29]. We performed a post-hoc analysis of this study to evaluate the potential clinical efficacy of panobacumab in the treatment of $P$. aeruginosa $\mathrm{O} 11$ nosocomial pneumonia. The 17 patients that received panobacumab were compared to a group of 14 patients screened in the study that did not receive immunotherapy.

\section{Patients and methods}

Study population

We performed a post-hoc analysis of the multicenter, open pilot phase IIa clinical trial (NCT00851435) which prospectively evaluated the safety and pharmacokinetics of at least one dose of $1.2 \mathrm{mg} / \mathrm{kg}$ panobacumab, a fully human monoclonal anti-LPS IgM, given every $72 \mathrm{~h}$ in 17 patients with microbiologically documented [bronchoalveolar lavage (BAL), mini-BAL] serotype O11 P. aeruginosa pneumonia [29]. Definitions and detailed inclusion/exclusion criteria are described elsewhere [29]. These patients (intent-to-treat group) and those receiving the full three-dose treatment (per-protocol group) were compared to a group consisting of patients who fulfilled all the inclusion and exclusion criteria of the phase IIa study but who could not receive panobacumab (not treated with panobacumab group) (Fig. 1) for the following reasons: (i) treatment refusal $(n=3)$; (ii) physicians refused to give the treatment $(n=3)$; (iii) patients were screened after the end of the trial $(n=3)$; (iv) delayed serotyping results $(n=2)$; or (iv) unknown reasons $(n=3)$.

Data collection

In addition to demographics (age, gender), the general condition of the patients was evaluated using the Acute Physiology and Chronic Health Evaluation II (APACHE II) score system [30]. Organ dysfunction was evaluated using the Sequential Organ Failure Assessment (SOFA) score [31]. The severity of pneumonia was assessed using the Clinical Pulmonary Infection Score (CPIS) [32].

\section{Clinical endpoints}

The clinical endpoints were as follows: (i) survival defined as survival by day 30 ; (ii) clinical resolution was defined as no persisting symptoms or complications attributable to the pneumonia with a return to normal values of all four of the following: core body temperature $\left(<38.3{ }^{\circ} \mathrm{C}\right)$, peripheral blood leucocyte count $\left(<10 \times 10^{3} / \mathrm{mm}^{3}\right), \mathrm{PaO}_{2} / \mathrm{FIO}_{2}$ ratio $(>25 \mathrm{kPa})$, and no or minimal growth of bacteria similar to those isolated from mini-BAL samples in quantitative cultures of endotracheal aspirates; (iii) time to clinical resolution was defined as free of pneumonia and recurrence (relapse caused by the same pathogen) at day 30 post-treatment; (iv) recurrence was defined as resolution of all clinical signs of pneumonia, including infiltrates, with a subsequent return of 
Fig. 1 Flow chart of patients included in the post-hoc analysis

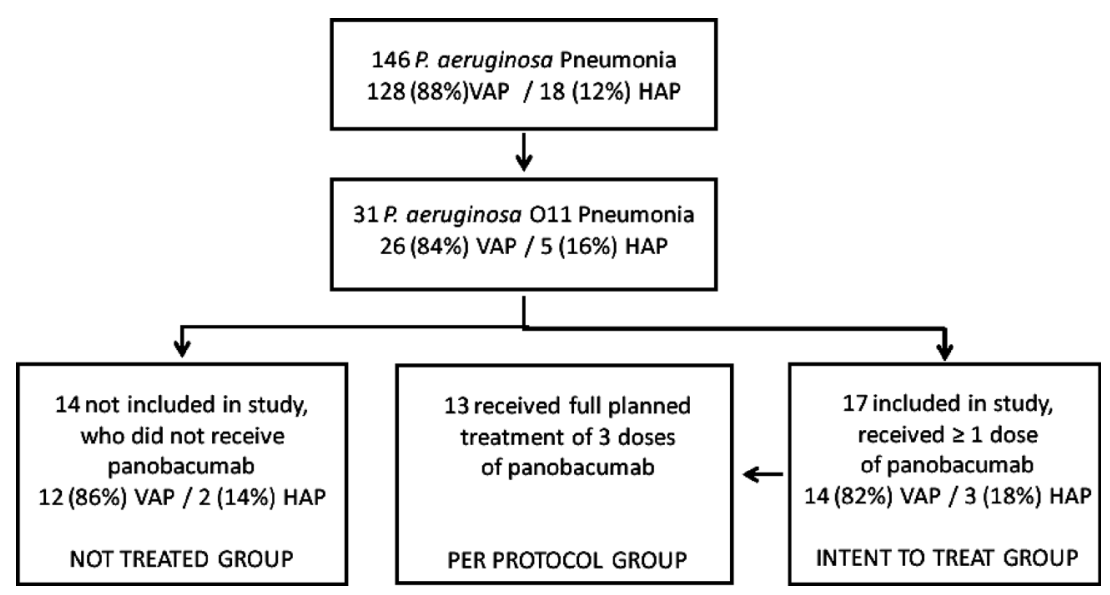

clinical signs and a diagnostic culture; (v) death was scored as a non-resolved infection.

Statistical analysis

Statistical analyses compared patients that received at least one dose or three doses of panobacumab to control patients. Continuous variables are presented as median [range, interquartile range (IQR)]. The characteristics of patients and clinical outcomes were compared using Fisher's exact test and the Wilcoxon rank-sum test, as indicated. Time to clinical resolution was analyzed using the Kaplan-Meier survival model. All tests were two-tailed, and statistical significance was established at $p$-values $<0.05$.

\section{Results}

Demographics and severity of nosocomial pneumonia

The phase IIa clinical trial designed to test the safety, pharmacokinetics, and potential efficacy of panobacumab adjunctive therapy screened $146 P$. aeruginosa microbiologically documented nosocomial pneumonia cases. Thirty-one patients were diagnosed with $\mathrm{O} 11$ serotype $P$. aeruginosa infections. Among these, 17 fulfilled the inclusion criteria (intent-to-treat group): four patients received only one dose and 13 patients received the planned three doses of panobacumab (perprotocol group). Fourteen patients suffering from nosocomial pneumonia caused by $P$. aeruginosa $\mathrm{O} 11$ but who did not receive panobacumab were used as untreated controls (Fig. 1).

The patient characteristics are summarized in Table 1. Compared to untreated patients, patients that received panobacumab presented with more severe forms of disease. These patients were older (72 [IQR: 64-79] versus 50 [IQR: 30-73] years on average; $p=0.024$ ) and had higher APACHE
II scores (17 [IQR: 16-22) versus 15 [IQR: 10-19]; $p=0.043$ ). The severity of pneumonia and the characteristics of antibiotic treatment were comparable among treated and untreated patients.

\section{Outcome}

The mortality rates were not statistically significant between groups. A total of six patients died, including three receiving only one dose of panobacumab. One patient died 3 days after the first doses from a gastrointestinal hemorrhage before receiving the second dose. Adjunctive treatment was stopped in a different patient after the first dose following worsening of preexisting cholestasis, who also presented with neutropenia and gastrointestinal bleeding (he died on day 17 from multiple organ failure). In a third patient, cardiac arrest followed by increased prothrombin time (possibly related to panobacumab treatment) precluded further panobacumab administration. This patient died on day 17 after a second cardiac arrest. Three patients who did not receive adjunctive treatment died on days 8,10 , and 16, respectively, from colitis-related septic shock, pneumonia-related multiple organ failure, and indeterminate causes, respectively.

\section{Effect of adjunctive passive immunotherapy}

When untreated patients were compared to patients that received $\geq 1$ dose of panobacumab (intent-to-treat group), there was no significant differences in clinical outcome, with a shorter time to clinical resolution of pneumonia and more disease-free days (Table 2). Differences were statistically significant when untreated patients were compared to patients that received the full planned treatment (three doses of panobacumab). Specifically, time to clinical resolution was 8.0 days (IQR: 7-12) versus 18.5 days (IQR: $8-30)(p=0.004)$, clinical resolution of 
Table 1 Characteristics of the patients presenting with Pseudomonas aeruginosa 011 nosocomial pneumonia

\begin{tabular}{|c|c|c|c|c|c|}
\hline & $\begin{array}{l}\text { All patients } \\
(n=31)\end{array}$ & $\begin{array}{l}\text { Not treated with } \\
\text { panobacumab } \\
(n=14)\end{array}$ & $\begin{array}{l}\text { Panobacumab } \\
\text { "intent-to-treat", } \geq 1 \\
\text { dose }(n=17)\end{array}$ & $\begin{array}{l}\text { Panobacumab } \\
\text { "per-protocol", } \\
\text { three doses }(n=13)\end{array}$ & $\begin{array}{l}p \text {-Values: not treated } \\
\text { vs. } \geq 1 \text { dose, not treated } \\
\text { vs. three doses }\end{array}$ \\
\hline Age (years), median (IQR) & $66(42-77)$ & $50(30-73)$ & $72(64-79)$ & $71(61-79)$ & $0.024, \mathrm{NS}$ \\
\hline Male/female (\%) & $70 / 30$ & $57 / 43$ & $82 / 18$ & $77 / 23$ & $0.039, \mathrm{NS}$ \\
\hline Initial antibiotic treatment, $n(\%)$ & & & & & NS, NS \\
\hline Bi-therapy & & $11(78.6 \%)$ & $10(58.8 \%)$ & $8(61.5 \%)$ & \\
\hline Beta-lactam + aminoglycoside & & 11 & 2 & 2 & \\
\hline Beta-lactam + quinolone & & & 6 & 5 & \\
\hline Beta-lactam + colistin & & & 2 & 1 & \\
\hline Monotherapy & & $3(21.4 \%)$ & $7(41.6 \%)$ & $5(38.5 \%)$ & \\
\hline Beta-lactam & & 3 & 6 & 4 & \\
\hline Quinolone & & & 1 & 1 & \\
\hline Inadequately treated with antibiotics, $n(\%)$ & $4(13.3 \%)$ & $1(7.1 \%)$ & $3(17.6 \%)$ & $2(15.5 \%)$ & NS, NS \\
\hline APACHE II score, median (IQR) & $17(14-20)$ & $15(10-19)$ & $17(16-22)$ & $17(15.5-23.5)$ & $0.043,0.048$ \\
\hline CPIS, median (IQR) & $8.0(7.0-9.0)$ & $8.0(5.8-9.3)$ & $9.0(7.5-9.5)$ & $9(8.0-9.5)$ & NS, NS \\
\hline SOFA score, median (IQR) & $6.0(4.5-9.0)$ & $7.0(4.8-9.3)$ & $6.0(4.0-7.0)$ & $5.5(3.8-7.8)$ & NS, NS \\
\hline $\begin{array}{l}\text { Length of hospital stay (days) before } \\
\text { pneumonia, median (IQR) }\end{array}$ & $15.0(7.0-29)$ & $14.5(4.8-32)$ & $15(8.5-32)$ & $17(8.5-36)$ & NS, NS \\
\hline $\begin{array}{l}\text { Length of ICU stay (days) before } \\
\text { pneumonia, (IQR) }\end{array}$ & $9.0(6.0-19.5)$ & $6.5(3.8-24)$ & $10.5(6.8-17.8)$ & $13(7.0-19.3)$ & $\mathrm{NS}, \mathrm{NS}$ \\
\hline
\end{tabular}

IQR interquartile range, NS not significant, CPIS Clinical Pulmonary Infection Score, APACHE II Acute Physiology and Chronic Health Evaluation II, SOFA Sequential Organ Failure Assessment

pneumonia at day 30 was $85 \%(11 / 13)$ in patients receiving three doses of panobacumab versus $64 \%$ $(9 / 14)$ in untreated patients $(p=0.048)$, and disease-free days were 22 (IQR: 18.5-23) versus 12.5 (IQR: 0-22) $(p=0.028)$. Kaplan-Meier survival curve analysis showed a statistically significantly shorter time to clinical resolution in patients receiving three doses of panobacumab (8.0 [IQR: 7.0-11.5] versus 18.5 [IQR: 8-30] days; $p=0.005$ ) (Fig. 2). Two out of 13 patients receiving three doses of panobacumab versus $1 / 14$ untreated patients developed recurrent pneumonia within 30 days (not statistically different).

\section{Discussion}

This manuscript describes a proof-of-concept analysis describing the administration of passive adjunctive immunotherapy of a human monoclonal antibody that specifically targeted

Table 2 Clinical outcomes

\begin{tabular}{|c|c|c|c|c|c|}
\hline & $\begin{array}{l}\text { All patients } \\
(n=31)\end{array}$ & $\begin{array}{l}\text { Not treated with } \\
\text { panobacumab } \\
(n=14)\end{array}$ & $\begin{array}{l}\text { Panobacumab } \\
\text { "intent-to-treat", } \geq 1 \\
\text { dose }(n=17)\end{array}$ & $\begin{array}{l}\text { Panobacumab } \\
\text { "per-protocol", } \\
\text { three doses }(n=13)\end{array}$ & $\begin{array}{l}p \text {-Values: not treated } \\
\text { vs. } \geq 1 \text { dose, not treated } \\
\text { vs. three doses }\end{array}$ \\
\hline $\begin{array}{l}\text { Time (days) to clinical resolution } \\
\text { of pneumonia, median (IQR) }\end{array}$ & $12.0(8.0-30)$ & $18.5(8.0-30)$ & $10.0(7.0-23)$ & $8.0(7.0-12)$ & NS, 0.004 \\
\hline Clinical resolution of pneumonia, $n(\%)$ & $20(65 \%)$ & $9(64 \%)$ & $11(65 \%)$ & $11(85 \%)$ & NS, 0.048 \\
\hline Disease-free days & $18(0-22)$ & $12.5(0-22)$ & $20(7.5-23)$ & $22(18.5-23)$ & NS, 0.028 \\
\hline Relapse within 30 days, $n(\%)$ & $4(13 \%)$ & $1(7 \%)$ & $3(18 \%)$ & $2(15 \%)$ & NS, NS \\
\hline Survival at day $30, n(\%)$ & $25(81 \%)$ & $11(79 \%)$ & $14(83 \%)$ & $13(100 \%)$ & NS, NS \\
\hline
\end{tabular}

$I Q R$ interquartile range 

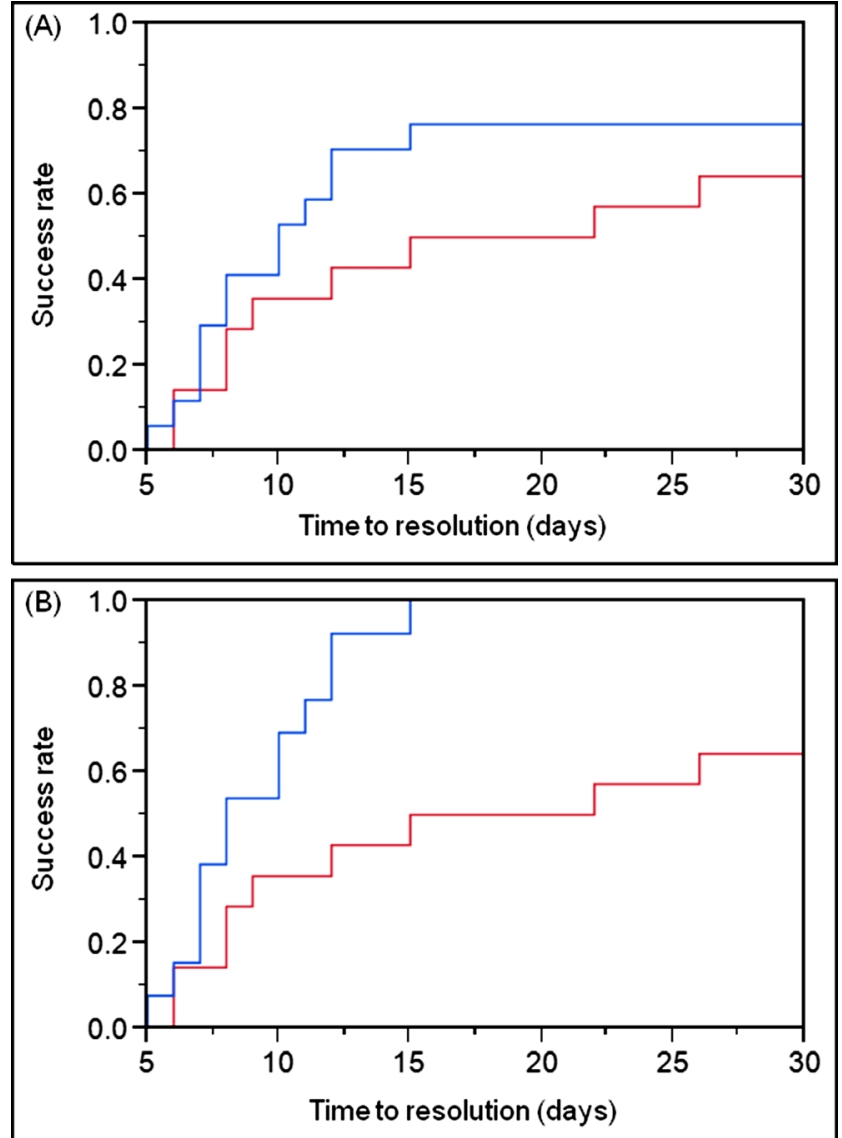

Fig. 2 Kaplan-Meier clinical resolution curve of patients receiving panobacumab versus untreated patients. a Patients with nosocomial Pseudomonas aeruginosa 011 pneumonia receiving $\geq 1$ dose of panobacumab (blue: intent-to-treat group; $n=17$ ) versus untreated patients (red: $n=14)$. $p$-Value (log-rank test): 0.3008 . b Patients with nosocomial $P$. aeruginosa $\mathrm{O} 11$ pneumonia receiving the full treatment (three doses) of panobacumab (blue: per-protocol group; $n=13$ ) versus untreated patients (red: $n=14)$. $p$-Value (log-rank test): 0.0045

the $P$. aeruginosa O11 LPS O-polysaccharide moiety. This form of immunotherapy may have improved the clinical outcome of patients presenting with nosocomial pneumonia.

Differences were statistically significant only between patients that received three doses of panobacumab. In these patients, an $85 \%$ success rate and a more rapid clinical resolution was achieved, despite these patients presenting with more severe infections and underlying conditions.

Three patients that received only one dose of panobacumab adjunctive immunotherapy died. One died early from septic shock related to the infection and two patients that were excluded by the independent phase IIa panobacumab clinical trial safety committee died later. This may be viewed as a limitation, but it should be pointed out that the full effect of adjunctive immunotherapy targeted at a virulence factor may not be achieved immediately and that repetitive doses may be required to achieve a clinical effect.

These results are consistent with previous in vitro and in vivo data obtained with panobacumab. The diffusion of panobacumab from blood into the alveolar spaces may have contributed to its efficacy [29]. Moreover, observed relapses at day 30 may be explained by the disappearance of panobacumab from the blood [29] at this time.

Conclusions regarding the clinical efficacy of panobacumab in the treatment of nosocomial $P$. aeruginosa $\mathrm{O} 11$ infections should be made with caution, since this post-hoc analysis consisted of a limited number of patients. Nevertheless, such preliminary results are encouraging and may justify a large prospective randomized clinical trial in critically ill patients presenting with $P$. aeruginosa nosocomial infections.

In summary, these preliminary results suggested that the full course of panobacumab adjunctive immunotherapy (three doses) targeting serotype-specific LPS of $P$. aeruginosa $\mathrm{O} 11$ may have improved the clinical outcome of patients presenting with nosocomial pneumonia.

Acknowledgments We thank Holger Koch, Verena Gafner, and Michael P. Rudolf from Kenta Biotech, Schlieren, Switzerland for the development of panobacumab. Now ARIDIS Pharmaceuticals, San Jose, USA.

Author contributions Y-AQ, J-LP, PE, and J-PR acquired patient data and prepared the manuscript.

J-PR performed the statistical analysis.

QL, J-JR, JC, MW, MT, EM, JG, BF, and P-FL acquired patient data.

AP, HL, and EM designed the study, and collected and analyzed the data from the original study.

Conflict of interest The following authors declare that they have no competing interests: Jorge Garbino, Qin Lu, Jean-Jacques Rouby, Emmanuelle Mercier, Michael Tamm, Michel Wolff.

The following authors were employees of Kenta Biotech at the time of the trial and owned stocks of Kenta Biotech: Holger Koch, Hedvika Lazar, Erkan Mus, Michael P. Rudolf.

Jean Chastre has received consulting and/or lecture fees from Kenta Biotech, Pfizer, Brahms, Astellas, KaloBios, Sanofi, Nektar-Bayer, and Glaxo-Smith-Kline.

Philippe Eggimann has received consulting and/or lecture fees from Kenta Biotech, Pfizer, Astellas, KaloBios, and MSD. He was involved as a consultant for other Kenta Biotech projects.

Bruno François has received consulting and/or lecture fees in the last 3 years from Kenta Biotech, Sanofi, Talecris, GSK, and MedImmune.

Verena Gafner is a former employee of Kenta Biotech.

Pierre-Francois Laterre is a consultant at Kenta Biotech, Agennix, and AstraZeneca.

Antonio Perez is external Chief Medical Officer for Kenta Biotech. Now ARIDIS, Clinical Development Advisor.

Funding The phase IIa study, but not this independent post-hoc analysis, was sponsored by Kenta Biotech AG. Now ARIDIS Pharmaceuticals, San Jose, USA. 


\section{References}

1. Eggimann P, Pittet D (2001) Infection control in the ICU. Chest 120(6):2059-2093

2. Vincent JL, Rello J, Marshall J, Silva E, Anzueto A, Martin CD, Moreno R, Lipman J, Gomersall C, Sakr Y, Reinhart K; EPIC II Group of Investigators (2009) International study of the prevalence and outcomes of infection in intensive care units. JAMA 302(21): 2323-2329

3. Tabah A, Koulenti D, Laupland K, Misset B, Valles J, Bruzzi de Carvalho F, Paiva JA, Cakar N, Ma X, Eggimann P, Antonelli M, Bonten MJ, Csomos A, Krueger WA, Mikstacki A, Lipman J, Depuydt P, Vesin A, Garrouste-Orgeas M, Zahar JR, Blot S, Carlet J, Brun-Buisson C, Martin C, Rello J, Dimopoulos G, Timsit JF (2012) Characteristics and determinants of outcome of hospitalacquired bloodstream infections in intensive care units: the EUROBACT International Cohort Study. Intensive Care Med 38(12):1930-1945

4. Harris AD, Johnson JK, Thom KA, Morgan DJ, McGregor JC, Ajao AO, Moore AC, Comer AC, Furuno JP (2011) Risk factors for development of intestinal colonization with imipenem-resistant Pseudomonas aeruginosa in the intensive care unit setting. Infect Control Hosp Epidemiol 32(7):719-722

5. Ong DS, Jongerden IP, Buiting AG, Leverstein-van Hall MA, Speelberg B, Kesecioglu J, Bonten MJ (2011) Antibiotic exposure and resistance development in Pseudomonas aeruginosa and Enterobacter species in intensive care units. Crit Care Med 39(11): 2458-2463

6. Kollef MH, Silver P, Murphy DM, Trovillion E (1995) The effect of late-onset ventilator-associated pneumonia in determining patient mortality. Chest 108(6):1655-1662

7. Trouillet JL, Vuagnat A, Combes A, Kassis N, Chastre J, Gibert C (2002) Pseudomonas aeruginosa ventilator-associated pneumonia: comparison of episodes due to piperacillin-resistant versus piperacillin-susceptible organisms. Clin Infect Dis 34(8):1047-1054

8. Parker CM, Kutsogiannis J, Muscedere J, Cook D, Dodek P, Day AG, Heyland DK; Canadian Critical Care Trials Group (2008) Ventilator-associated pneumonia caused by multidrug-resistant organisms or Pseudomonas aeruginosa: prevalence, incidence, risk factors, and outcomes. J Crit Care 23(1):18-26

9. Peña C, Gómez-Zorrilla S, Oriol I, Tubau F, Dominguez MA, Pujol M, Ariza J (2013) Impact of multidrug resistance on Pseudomonas aeruginosa ventilator-associated pneumonia outcome: predictors of early and crude mortality. Eur J Clin Microbiol Infect Dis 32(3):413-420

10. Boucher HW, Talbot GH, Benjamin DK Jr, Bradley J, Guidos RJ, Jones RN, Murray BE, Bonomo RA, Gilbert D; Infectious Diseases Society of America (2013) $10 \times$ '20 Progress - development of new drugs active against gram-negative bacilli: an update from the Infectious Diseases Society of America. Clin Infect Dis 56(12): 1685-1694

11. Chastre J, Luyt CE (2010) Other therapeutic modalities and practices: implications for clinical trials of hospital-acquired or ventilatorassociated pneumonia. Clin Infect Dis 51(Suppl 1):S54-S58

12. François B, Luyt CE, Dugard A, Wolff M, Diehl JL, Jaber S, Forel JM, Garot D, Kipnis E, Mebazaa A, Misset B, Andremont A, Ploy MC, Jacobs A, Yarranton G, Pearce T, Fagon JY, Chastre J (2012) Safety and pharmacokinetics of an anti-PcrV PEGylated monoclonal antibody fragment in mechanically ventilated patients colonized with Pseudomonas aeruginosa: a randomized, double-blind, placebocontrolled trial. Crit Care Med 40(8):2320-2326

13. Hsu JL, Safdar N (2011) Polyclonal immunoglobulins and hyperimmune globulins in prevention and management of infectious diseases. Infect Dis Clin North Am 25(4):773-788

14. [No authors listed] (1992) Prophylactic intravenous administration of standard immune globulin as compared with core-lipopolysaccharide immune globulin in patients at high risk of postsurgical infection. The Intravenous Immunoglobulin Collaborative Study Group. N Engl J Med 327(4):234-240

15. Laupland KB, Kirkpatrick AW, Delaney A (2007) Polyclonal intravenous immunoglobulin for the treatment of severe sepsis and septic shock in critically ill adults: a systematic review and meta-analysis. Crit Care Med 35(12):2686-2692

16. Abraham E (1998) Cytokine modifiers: pipe dream or reality? Chest 113(3 Suppl):224S-227S

17. Ter Meulen J (2011) Monoclonal antibodies in infectious diseases: clinical pipeline in 2011. Infect Dis Clin North Am 25(4):789-802

18. Rotschild M, Elias N, Berkowitz D, Pollak S, Shinawi M, Beck R, Bentur L (2005) Autoantibodies against bactericidal/permeabilityincreasing protein (BPI-ANCA) in cystic fibrosis patients treated with azithromycin. Clin Exp Med 5(2):80-85

19. Sawada S, Kawamura T, Masuho Y (1987) Immunoprotective human monoclonal antibodies against five major serotypes of Pseudomonas aeruginosa. J Gen Microbiol 133(12):3581-3590

20. Lang AB, Schaad UB, Rüdeberg A, Wedgwood J, Que JU, Fürer E, Cryz SJ Jr (1995) Effect of high-affinity anti-Pseudomonas aeruginosa lipopolysaccharide antibodies induced by immunization on the rate of Pseudomonas aeruginosa infection in patients with cystic fibrosis. J Pediatr 127(5):711-717

21. Hemachandra S, Kamboj K, Copfer J, Pier G, Green LL, Schreiber JR (2001) Human monoclonal antibodies against Pseudomonas aeruginosa lipopolysaccharide derived from transgenic mice containing megabase human immunoglobulin loci are opsonic and protective against fatal pseudomonas sepsis. Infect Immun 69(4): 2223-2229

22. Horn MP, Zuercher AW, Imboden MA, Rudolf MP, Lazar H, Wu H, Hoiby N, Fas SC, Lang AB (2010) Preclinical in vitro and in vivo characterization of the fully human monoclonal IgM antibody KBPA101 specific for Pseudomonas aeruginosa serotype IATSO11. Antimicrob Agents Chemother 54(6):2338-2344

23. Secher T, Fauconnier L, Szade A, Rutschi O, Fas SC, Ryffel B, Rudolf MP (2011) Anti-Pseudomonas aeruginosa serotype O11 LPS immunoglobulin M monoclonal antibody panobacumab (KBPA101) confers protection in a murine model of acute lung infection. J Antimicrob Chemother 66(5):1100-1109

24. Hostacká A, Majtán V (1997) Serotyping and virulence factors of Pseudomonas aeruginosa clinical isolates. Acta Microbiol Immunol Hung 44(2):141-146

25. Faure K, Shimabukuro D, Ajayi T, Allmond LR, Sawa T, WienerKronish JP (2003) O-antigen serotypes and type III secretory toxins in clinical isolates of Pseudomonas aeruginosa. J Clin Microbiol 41(5):2158-2160

26. Fonseca AP, Correia P, Sousa JC, Tenreiro R (2007) Association patterns of Pseudomonas aeruginosa clinical isolates as revealed by virulence traits, antibiotic resistance, serotype and genotype. FEMS Immunol Med Microbiol 51(3):505-516

27. Le Berre R, Nguyen S, Nowak E, Kipnis E, Pierre M, Quenee L, Ader F, Lancel S, Courcol R, Guery BP, Faure K; Pyopneumagen Group (2011) Relative contribution of three main virulence factors in Pseudomonas aeruginosa pneumonia. Crit Care Med 39(9):2113-2120

28. Lu Q, Eggimann P, Luyt CE, Wolff M, Tamm M, François B, Mercier E, Garbino J, Laterre PF, Koch H, Gafner V, Rudolf MP, Mus E, Perez A, Lazar H, Chastre J, Rouby JJ (2014) Pseudomonas aeruginosa serotypes in nosocomial pneumonia: prevalence and clinical outcomes. Crit Care 18(1):R17

29. Lu Q, Rouby JJ, Laterre PF, Eggimann P, Dugard A, GiamarellosBourboulis EJ, Mercier E, Garbino J, Luyt CE, Chastre J, GeorgescuKyburz V, Rudolf MP, Gafner V, Lazar H, Koch H, Perez A, Krämer SD, Tamm M (2011) Pharmacokinetics and safety of panobacumab: specific adjunctive immunotherapy in critical patients with nosocomial Pseudomonas aeruginosa O11 pneumonia. J Antimicrob Chemother 66(5):1110-1116 
30. Knaus WA, Draper EA, Wagner DP, Zimmerman JE (1985) APACHE II: a severity of disease classification system. Crit Care Med 13(10):818-829

31. Vincent JL, Moreno R, Takala J, Willatts S, De Mendonça A, Bruining H, Reinhart CK, Suter PM, Thijs LG (1996) The SOFA (Sepsis-related Organ Failure Assessment) score to describe organ dysfunction/failure. On behalf of the Working Group on Sepsis-
Related Problems of the European Society of Intensive Care Medicine. Intensive Care Med 22(7):707-710

32. Luna CM, Blanzaco D, Niederman MS, Matarucco W, Baredes NC, Desmery P, Palizas F, Menga G, Rios F, Apezteguia C (2003) Resolution of ventilator-associated pneumonia: prospective evaluation of the clinical pulmonary infection score as an early clinical predictor of outcome. Crit Care Med 31(3):676-682 